\title{
Chemical Bonding Information from ELNES using Bond Overlap Population Diagram
}

\author{
Teruyasu Mizoguchi ${ }^{1,2}$ and Isao Tanaka ${ }^{3}$ \\ ${ }^{1}$ National Center for Electron Microscopy, Lawrence Berkeley National Laboratory, One Cyclotron \\ road, Berkeley, CA 94720, USA \\ ${ }^{2}$ Institute of Engineering Innovation, The University of Tokyo, 2-11-16, Yayoi, Bunkyo, Tokyo \\ 116-0013, Japan \\ ${ }^{3}$ Department of Materials Science and Engineering, Kyoto University, Sakyo, Kyoto 606-8501 \\ Japan.
}

ELNES reflects the electron transition from a core-orbital to an unoccupied band. The ELNES therefore has the chemical bonding information around an objective atom. In order to reproduce and interpret the experimental ELNES, the first principles calculation of ELNES has been extensively performed. [1-13] Recently, some of theoretical studies succeeded in quantitative reproduction of the experimental spectra.[7-13] However, the relationships between spectral features and the chemical information has not been clarified in detail, even when experimental spectra are well reproduced by the calculations.

We have reported that the bond overlap population (BOP) diagram is useful for detailed analysis of the ELNES.[5,6] The BOP diagram has been computed by broadening of BOP at each energy level obtained by a first principles molecular orbital method using a cluster. For crystalline systems, calculations imposing periodic boundary condition should be a natural way. In this study, we report the BOP diagram obtained by a first principles band structure method.

First principles band structure calculation using orthogonalized linear combination of atomic orbitals (OLCAO) method is employed.[14] In this method, atomic orbitals, which are expressed by the sum of Gaussian functions, are used as basis functions. Therefore, chemical bonding information, such as BOP and net charge can be calculated in a straightforward manner. Figure 1 shows the experimental and theoretical Mg-K edge ELNES together with the BOP diagram. The calculation has been made using 128 atom-supercell, and a core-hole was introduced at a Mg-1s orbital. Since Mg-K edge ELNES reflects the electronic transition to unoccupied Mg-p orbitals, the BOP diagram between Mg-p orbital and the surrounding O-s, p and Mg-s, p, d orbitals are shown. Positive and negative values of BOP respectively corresponds to the bonding and antibonding interaction. The overlap populations among $\mathrm{Mg}^{*}$ and $\mathrm{O}$ orbitals mainly exhibit an antibonding interaction. On the other hand, the $\mathrm{Mg}^{*}-\mathrm{Mg}$ interactions show both bonding and antibonding interactions. The shape of the wave functions in the unoccupied band is therefore well characterized by the $\mathrm{Mg}^{*}-\mathrm{Mg}$ interaction. The peaks $\mathrm{A}$ and $\mathrm{D}$ are determined by the bonding and antibonding interactions of 
Mg*p-Mg s. The peaks B and E can be mainly ascribed to the bonding and antibonding interactions of $\mathrm{Mg}^{*} \mathrm{p}-\mathrm{Mg}$ s. The broad peak around $\mathrm{F}$ can be due to antibonding interactions of $\mathrm{Mg}^{*} \mathrm{p}-\mathrm{Mg} \mathrm{d}$.

Origin of ELNES peaks can be interpreted in this way using BOP diagrams. BOP diagrams of $\mathrm{AlN}, \mathrm{GaN}, \mathrm{InN}, \mathrm{SiC}, \mathrm{ZnO}$ and their polymorphs are also discussed.
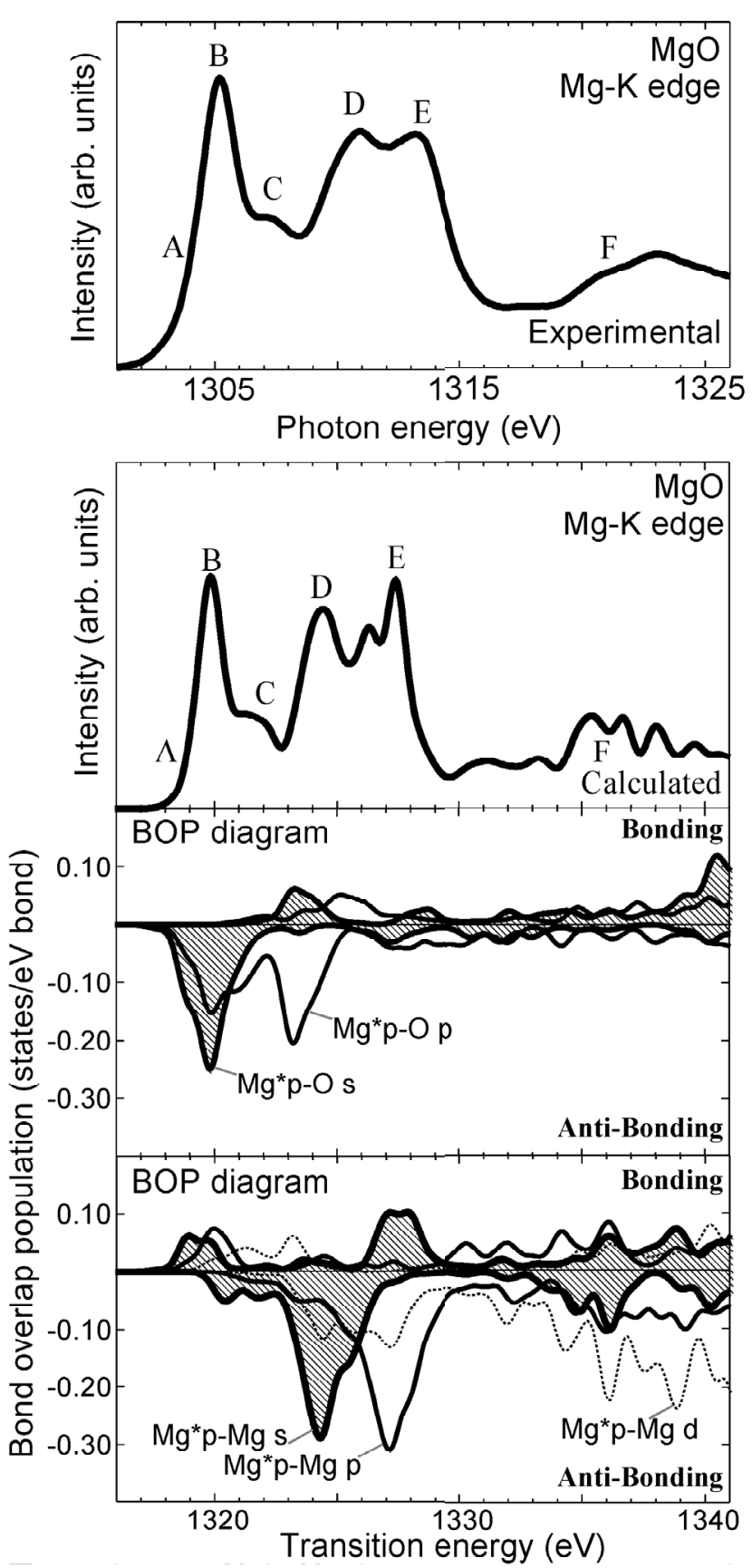

Fig. 1 (top to bottom) Experimental and theoretical $\mathrm{Mg}-\mathrm{K}$ edge and the BOP diagram between $\mathrm{Mg}^{*}$ and surrounding $\mathrm{O}$ and $\mathrm{Mg}$. $\mathrm{Mg}^{*}$ represents the $\mathrm{Mg}$ atom which includes a core-hole.

\section{References}

[1] I. Tanaka et al. Phys. Rev. B 54, 4604 (1996).

[2] S. Köstlemeier et al. Phys. Rev. B 60, 14025 (1999).

[3] P. Rez et al. Ultramicroscopy 78, 175 (1999).

[4] K. Lie et.al. Phys. Rev. B 59, 5361 (1999).

[5] I. Tanaka et al. Phys. Rev B 60, 4944 (1999).

[6] T. Mizoguchi et a.. Phys. Rev. B 61, 2180 (2000).

[7] S. D. Mo et al. Phys. Rev. B 62, 7901 (2000).

[8] C. Hébert et al., Micron 34. 219 (2003).

[9] K. Schwarz et al., Comp. Mat. Sci. 28, 259 (2003).

[10] K. van Benthem et al., Ultramicroscopy, 96509 (2003).

[11]T. Mizoguchi et al. Phys. Rev. B. 70045103 (2004).

[12] S-P. Gao et al., Appl. Phys. Lett., 84, 2784 (2004).

[13] I. Tanaka et al. J. Am. Ceram. Soc. (Feature article) submitted.

[14] W. Y. Ching, J. Am. Ceram. Soc. 73, 3135 (1990).

[15] The authors thank Wai Yim Ching for allowing us to use the OLCAO program. T. M is supported as Japan Society for Promotion of Science (JSPS) fellow. This work is supported by a Grant-in-Aid for General Scientific Research from the Ministry of Education, Sports, Science and Culture of Japan. 